Mappemonde

Revue trimestrielle sur l'image géographique et les formes du territoire

$121 \mid 2017$

Varia

\title{
Anticiper la recomposition du territoire
}

\section{Annabelle Moatty}

\section{OpenEdition}

\section{Journals}

Édition électronique

URL : http://journals.openedition.org/mappemonde/3766

DOI : 10.4000/mappemonde.3766

ISSN : 1769-7298

Éditeur

UMR ESPACE

\section{Référence électronique}

Annabelle Moatty, «Anticiper la recomposition du territoire », Mappemonde [En ligne], 121 | 2017, mis en ligne le 01 juillet 2017, consulté le 15 septembre 2020. URL : http://journals.openedition.org/ mappemonde/3766

Ce document a été généré automatiquement le 15 septembre 2020.

\section{(c) (i) (2)(2)}

La revue Mappemonde est mise à disposition selon les termes de la Licence Creative Commons Attribution - Pas d'Utilisation Commerciale - Partage dans les Mêmes Conditions 4.0 International. 


\title{
Anticiper la recomposition $\mathrm{du}$ territoire
}

\author{
Annabelle Moatty
}

\author{
Références de la thèse \\ MOАТTY А. (2015). Pour une géographie des reconstructions post-catastrophe : risques, \\ sociétés et territoires. Thèse de géographie, Université Paul Valéry - Montpellier 3
}

1 Après une catastrophe, la rapidité et le succès du relèvement et de la reconstruction dépendent de la manière dont sont articulées politique et géographie (Alexander, 2010). Se dessine en filigrane la nécessité de prendre en compte le fonctionnement réticulaire et multiscalaire des sociétés et de leurs territoires. Anticiper la recomposition du territoire est fort complexe et comporte beaucoup d'incertitudes en lien direct avec son caractère dynamique et évolutif. La difFiculté réside aussi dans l'intégration des modes de relation et d'interdépendance entre les territoires pour envisager leur recomposition (Perrow, 2005, cité par Comfort et al., 2010). Plusieurs prérequis s'imposent pour anticiper les recompositions territoriales, comme la connaissance et la maîtrise du foncier et des lois qui régissent sa gestion par exemple. Les pays ne sont pas égaux dans la connaissance et la maîtrise du foncier. Aussi, il est nécessaire d'anticiper la mise à jour du cadastre lorsqu'il est lacunaire ou inexistant (El Anwar et al., 2009). Cela implique aussi de concevoir des montages administratifs qui facilitent la reconversion des territoires et le redéveloppement en dehors des zones à risque. Cependant, il n'est pas toujours possible d'utiliser les ressources locales lorsqu'elles sont « mal localisées » car trop loin des bassins économiques et des aires de vie.

2 Toutes les adaptations réalisées pour recomposer le territoire ne sont pas nécessairement en phase avec les prérogatives de la prévention et peuvent même être contradictoires. Ainsi, dans le Var, on a vu des bâtiments être construits dans des zones inondées en juin 2010. La raison invoquée était que les permis de construire avaient été attribués avant la catastrophe, pour autant aucune modification préventive n'a été apportée aux permis. L'autre raison était le droit à la densification de l'urbanisation qui peut être la seule possibilité de poursuivre l'urbanisation. «La gestion préventive des 
catastrophes favorise l'intensification de l'urbanisation, recomposée, précisément parce qu'elle ne permet jamais d'éliminer totalement les risques » (Pigeon, 2010, p. 13).

Nous avons évoqué ici les problématiques des recompositions territoriales à moyen terme, mais certains éléments appartenant à la post-crise immédiate et à la restauration peuvent aussi être anticipés. Maret et Cadoul (2008) mettent en avant le rôle majeur de la topographie dans la durée de la crise à la Nouvelle-Orléans. La ville est située dans une cuvette qui emprisonne les eaux, le seul moyen de les évacuer est d'utiliser des moyens mécaniques. Ce type de mesure s'appliquera aussi aux inondations et submersions en présence de digues ou encore de lits en toit. Ce temps est aussi celui de la gestion des débris. Leur gestion pose de nombreux problèmes, sanitaires d'abord et financiers car elle peut engendrer des surcoûts importants. L'anticipation de la gestion des déchets pour faciliter le nettoyage et l'évaluation des dommages - et ainsi pouvoir envisager la reprise d'activité plus rapidement - passe par le développement d'ententes avec les localités voisines pour organiser le déblaiement, le stockage et la destruction. Par la suite, on peut imaginer l'élaboration d'un programme de recyclage, notamment en choisissant à l'avance des sites de stockage et recyclage (McEntire, 2007).

La figure ci-après présente une synthèse des "contraintes territoriales » qui restreignent les possibilités de recomposer le territoire de manière préventive en période de reconstruction. Le cas de figure représenté ici s'inspire de nos terrains indonésiens et plus précisément des villages de la province de Java Centre autour des problématiques de délocalisation et relocalisation. Le territoire sinistré (en gris foncé) est localisé à proximité d'un axe routier majeur puisqu'il dessert le pôle attractif de la région : la ville la plus proche. La proximité de cet axe est un atout pour ce territoire puisqu'elle permet aux communautés d'accéder rapidement et aisément aux services et infrastructures de santé, d'éducation, et aux commerces. De fait, les terrains qui jouxtent cet axe sont très prisés. De plus, de part et d'autre de la route les terres agricoles sont particulièrement fertiles et productives. Du point de vue des communautés qui doivent être relocalisées, il est donc préférable de voir le centre de relogement s'implanter à l'ouest de leur village d'origine (cf. figure 1). Cependant, pour les communautés d'accueil, vendre ces terres agricoles n'est pas une solution durable ni viable car ils se sépareraient de leur source principale de revenus. De plus, à l'échelle du bassin de vie, la vente de ces terres fertiles pour y construire les centres de relogement peut contribuer à déstabiliser l'économie locale puisque ces agriculteurs devront soit se reconvertir, soit acheter de nouvelles terres plus éloignées de leurs lieux de vie du fait de la pression foncière et de l'absence de parcelles cultivables à proximité. 
Figure 1. Représentation schématique des contraintes territoriales dans le processus de reconstruction

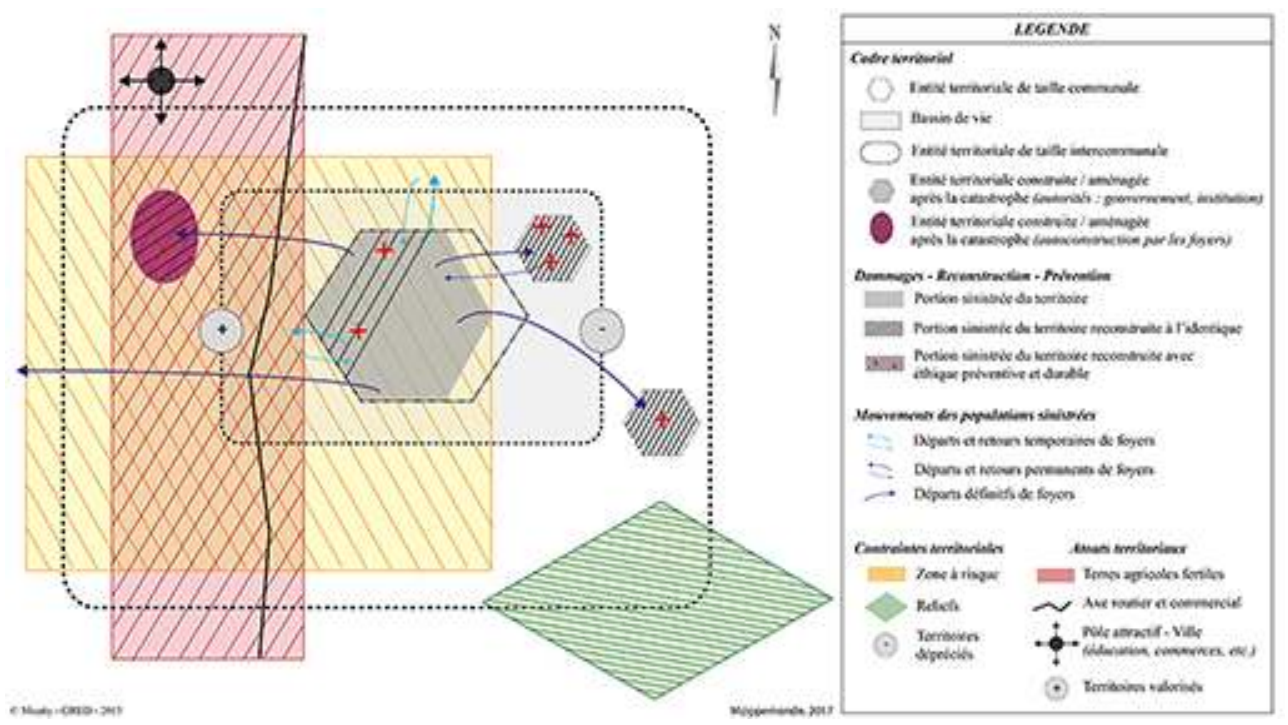

S'ajoute à cette équation le zonage des risques présents sur le territoire. Les terres qui sont les plus attractives pour les communautés à déplacer sont en partie localisées en zone à risque et les autorités ne peuvent pas assumer de délocaliser et relocaliser des foyers là où il interdit la construction. Les éléments topographiques sont aussi à prendre en compte puisqu'ils contraignent la construction, ou du moins la compliquent et en augmentent les coûts. Les parcelles pressenties pour l'implantation des centres de relogement gouvernementaux se limitent donc au quart nord-est du bassin de vie, et à l'est du groupement de communes, là où les enjeux économiques sont moindres (cf. figure 1). Les foyers doivent donc accepter de partir pour une nouvelle localité, moins attractive parce que disposant de moins de ressources.

Face à ce choix, certains choisiront l'auto construction pour limiter l'éloignement avec leurs sources de revenus, les services et infrastructures de la ville, et avec leurs villages d'origine. Ces foyers, qui sont en capacité de se relocaliser sans l'aide financière du gouvernement, sont en majorité issus de la classe moyenne. On compte aussi quelques foyers plus modestes qui bénéficient de l'aide de leurs familles, qui opèrent une stratégie de rapprochement familial et quittent définitivement le village. Pour les autres foyers, trois catégories se distinguent. Premièrement, il y a ceux qui resteront au village (par peur de ne pas s'adapter dans les centres, de ne pas retrouver de travail, par attachement à leur terre, etc.) et qui reconstruiront leur maison sur place avec éventuellement quelques améliorations préventives marginales (emploi de matériaux plus résistants pour refaire un mur détruit par exemple). Deuxièmement, il y a les foyers dont la maison a été totalement détruite et qui sont entièrement dépendants des programmes de relogement du gouvernement. Ces ménages, prioritaires dans le processus de relogement ont obtenu une place dans le centre le plus proche du village, avec lequel ils continuent à entretenir des relations. Enfin, troisièmement, il y a les foyers qui souhaitent quitter définitivement le village à cause du traumatisme laissé par la catastrophe mais dont la maison n'a pas été détruite. Leur relogement a pris plus de temps et s'est efFectué dans un centre plus éloigné du village.

7 L'objectif de l'anticipation est donc de concevoir des projets à moyen et long terme autours desquels toutes les parties prenantes sont rassemblées (même si le consensus 
reste utopique au regard des intérêts divergents) autour de l'intérêt général pour donner du poids aux décisions allant dans le sens d'une «éthique préventive » et de la durabilité.

\section{BIBLIOGRAPHIE}

ALEXANDER D. (2010). « Post disaster reconstruction: planning and sustainability ». Université de Florence, $35 \mathrm{p}$.

COMFORT L.K., SICILIANO M.D., OKADA A. (2010). « Risque, résilience et reconstruction : le tremblement de terre haïtien du 12 janvier 2010 ». Télescope, vol. 16, n² 2, p. 37-58.

EL ANWAR O., EL-RAYES K., ELNASHAI A. (2009). « An automated system for optimizing post-disaster temporary housing allocation, Automation in Construction ». Automation in Construction, vol. 18, $\mathrm{n}^{\circ} 7$ p. 983-993.

MARET I., CADOUL T. (2008). « Résilience et reconstruction durable : que nous apprend La NouvelleOrléans?». Annales de géographie, n 663, p. 104-124.

MCENTIRE, D. (2007). Disaster Response and Recovery. Hoboken, NJ: Wiley, 498 p. ISBN 0471789747

PERROW C. (2005). Organizing America: Wealth, Power, and the Origins of Capitalism. Princeton University Press, 272 p. ISBN 9780691123158

PIGEON P. (2010). «Catastrophes dites naturelles, risques et développement durable : Utilisations géographiques de la courbe de Farmer ». VertigO, vol. 10, n 1. DOI 10.4000/vertigo.9491. https:// journals.openedition.org/vertigo/9491

\section{INDEX}

Mots-clés : prix de thèse, prix de thèse 2016 